Published in Appl. Phys. Lett. 84, 227 (2004).

\title{
Cross-sectional Scanning Tunneling Microscopy and Spectroscopy of InGaP/GaAs Heterojunctions
}

\author{
Y. Dong and R. M. Feenstra \\ Dept. Physics, Carnegie Mellon University, Pittsburgh, PA 15213 \\ M. P. Semtsiv and W. T. Masselink \\ Department of Physics, Humboldt-Universität zu Berlin, D-10115 Berlin, Germany
}

\begin{abstract}
Compositionally abrupt InGaP/GaAs heterojunctions grown by gas-source molecular beam epitaxy have been investigated by cross-sectional scanning tunneling microscopy and spectroscopy. Images inside the $\mathrm{InGaP}$ layer show non-uniform In and $\mathrm{Ga}$ distribution. About $1.5 \mathrm{~nm}$ of transition region at the interfaces is observed, with indium carryover identified at the GaAs-on-InGaP interface. Spatially resolved tunneling spectra with nanometer spacing across the interface were acquired, from which band offsets (revealing that nearly all of band offset occurs in the valence band) were determined.
\end{abstract}

Heterojunctions between $\operatorname{In}_{\mathrm{x}} \mathrm{Ga}_{1-\mathrm{x}} \mathrm{P}$ (hereafter $\mathrm{InGaP}$ ) and $\mathrm{GaAs}$ have attracted attention recently because of their device applications. It has been predicted and confirmed that this system should have a large fraction of the total energy gap discontinuity occurring in the valence band, which improves the electron injection efficiency of an heterojunction bipolar transistor (HBT) [1]. However, there exist discrepancies between band offsets results obtained by different techniques for samples grown by different methods/groups. It is known that properties of the interface depend sensitively on the detailed growth conditions [2-6]. Prior experimental techniques used in this system are not spatially resolved and in order to better understand this material system a study of the atomic-scale structural and electronic properties of the junctions is useful.

In this work we use scanning tunneling spectroscopy (STS) to study the spatial variation of the $\mathrm{InGaP} / \mathrm{GaAs}$ electronic band structure at nanometer length scales. The advantage of such work is that band offsets can be measured while simultaneously imaging the atomic-scale structural properties of the interfaces. Due to the experimental challenges of measuring band offsets by STS, there are only a few prior examples of such work, most notably on AlGaAs/GaAs [7]. Liu et al. have used cross-sectional STM to study the ordering effect of InGaP [8]. In this paper, we report cross-sectional STM and STS studies of compositionally abrupt InGaP/GaAs heterojunctions prepared by gassource molecular beam epitaxy (GSMBE). Images inside the InGaP layer reveal a random arrangement of In and Ga atom. This result is consistent with PL results and growth conditions for similar samples that indicate a nearly fully disordered InGaP layer [9]. It is found that GaAs-on-InGaP interface has a slightly wider transition region and more interface intermixing than the InGaP-on-GaAs interface. Both interfaces exhibit InGaAs-like properties. Indium outdiffusion from InGaP into GaAs at the GaAs-on- 
InGaP interface is clearly identified, although $\mathrm{As} / \mathrm{P}$ interchange is not very obvious in this study. Spatially resolved spectra reveal that nearly all of the band gap discontinuity occurs between the valence band edges.

A multilayer structure $\mathrm{p}-\mathrm{GaAs} / \mathrm{i}-\mathrm{InGaP} / \mathrm{p}-\mathrm{GaAs}$ was grown on a $\mathrm{p}$-type $\mathrm{GaAs}(001)$ substrate using GSMBE. The $\operatorname{In}_{\mathrm{x}} \mathrm{Ga}_{1-\mathrm{x}} \mathrm{P}$ was lattice matched to GaAs, with $\mathrm{x} \approx 0.485$ (growth temperature is $430^{\circ} \mathrm{C}$ ). GaAs layers are doped with Be at $1 \times 10^{18} \mathrm{~cm}^{-3}$, while the InGaP is not intentionally doped. The first GaAs layer growth is terminated with a $60 \mathrm{~s}$ purge of As. After $30 \mathrm{~s}$ venting, a short $3 \mathrm{~s}$ P exposure precedes the InGaP growth. The InGaP layer, nominally $50 \mathrm{~nm}$ thick, is then grown, terminated with a $30 \mathrm{~s}$ P soak. Then, after $1 \mathrm{~s}$ venting, $5 \mathrm{~s}$ As exposure precedes the growth of the second layer GaAs. An atomically flat $(1 \overline{1} 0)$ surface was exposed by cleavage in the STM chamber, under a background pressure of less than $5 \times 10^{-11}$ Torr. Commercial Pt-Ir probe tips are used. Topographic imaging is performed at a constant current of $0.1 \mathrm{nA}$ and at sample voltages specified below. For spectroscopic measurements, a large dynamic range of currents was achieved by varying the tip-sample separation [10]. One measures the tunneling current $I$, and differential conductance $d I / d V$, as a function of the sample voltage $V$. Then a normalized conductance $(d I / d V) / \overline{(I / V)}$ is computed, where some broadening is applied to $I / V$ (we use $1.5 \mathrm{~V}$ ) to produce a suitable normalization quantity [10].

In the large-scale STM image of Fig. 1(a) the InGaP layer is seen in the center part of the image with GaAs layers seen on either side. Growth direction is from the right to the left for all images presented in this paper. The InGaP layer appears mottled due to compositional fluctuations in the alloy. A high-resolution image of the InGaP layer is shown in Fig. 1(b). At a sample bias of $-2.0 \mathrm{~V}$, filled states are imaged, i.e. localized on P atoms for InGaP. The pattern of different brightness for the $\mathrm{P}$ atoms reflects the distribution of neighboring In and Ga atoms. Because the cleaved surface is atomically flat, the observed contrast arises from a combination of electronic and strain effects, both associated with the presence in the alloy of clusters that are InP-rich or GaP-rich [11]. Ordering of the alloy is an important phenomenon for InGaP. It has been found that, under certain growth conditions, the cations (Ga and In) are ordered on ( $\overline{1} 11)$ or $(1 \overline{1} 1)$ planes $[8,12]$. From the STM images here, the InGaP layer does not display obvious ordering. Previous studies reveal that the 4K PL emission will change from $1.98 \mathrm{eV}$ to $1.91 \mathrm{eV}$ when InGaP layer goes from fully disordered to partially ordered [12]. PL spectra from an InGaP sample with identical growth condition to the sample used here shows a peak energy of $1.97 \mathrm{eV}$ [9]. It is thus determined that the InGaP layer studied here is very close to fully disordered.

Figures 2(a) and (b) show high-resolution images of inverted and normal $\mathrm{InGaP} / \mathrm{GaAs}$ interfaces, respectively. Atoms on the group $\mathrm{V}$ sublattice are imaged here, i.e. revealing As atoms in GaAs and $\mathrm{P}$ atoms in InGaP. Arrows indicate the nominal position of the interfaces. The two interfaces display different features. For the GaAs-onInGaP interface, the transition region is about 3-4 atomic bilayers (bilayer spacing is 5.65 $\AA$ ) and most of it lies in the GaAs side. Some atom-size bright features are seen in the GaAs layer near the interface. We attribute these features to In atoms. The image of In atoms will appear brighter because In atoms are bigger than Ga atoms (also the band gap of InAs is smaller than that of GaAs, which would contribute to a larger tunnel current near In atoms [11]). Indium is known to segregate on InGaP surface [13,14], and thus it will likely incorporate into following GaAs layer. It was proposed that the exchange of $\mathrm{P}$ 
atoms by As atoms is the main reason for degradation of interface abruptness, especially for GaAs-on-InGaP interfaces [6]. Since the sizes of As atoms and $\mathrm{P}$ atoms along with the electronic structure of As-related and P-related compounds are different, the appearances of these atoms would be expected to be different in STM images. However it is difficult to observe As atoms inside the InGaP layer since this layer is disordered (appearing as a mottled image). Concerning $\mathrm{P}$ atoms on GaAs side, we would expect them to produce dark atomic-site features, but we do not observe a significant number of such features. We conclude that As/P interchange is not predominant at this interface.

Turning to the InGaP-on-GaAs interface, Fig. 2(b), the intermixing of atoms across this interface is not obvious. Large white features appearing in the image are cleavageinduced defects or residual surface contamination. A single atomic line just on the interface does appear brighter compared with its neighboring lines. That atomic line is probably InGaAs-like (a GaP-like interface would look dark). This result is consistent with our expectation from the growth conditions: The first GaAs layer growth is terminated with a $60 \mathrm{~s}$ purge of As. After $30 \mathrm{~s}$ venting, a short $3 \mathrm{~s} \mathrm{P}$ exposure precedes the InGaP growth. It is unlikely that $\mathrm{P}$ atoms will replace As atoms during this time. This InGaP-on-GaAs interface is thus likely to be InGaAs-like rather than GaP-like.

Fig. 3(A) is an atomic-resolution image of the GaAs-on-InGaP interface. A vertical dashed white line defines the nominal interface, as apparent in the image. Eight white filled circles, labeled (a)-(h), represent the position where the spectra in Fig. 3(B) were acquired. The conduction band minimum (CBM) and the valence band maximum (VBM) have been indicated using dashed lines, determined as described in Ref [10] with an accuracy of \pm 0.05 V. Spectrum (a) of Fig. 3 was acquired inside the GaAs layer, $1.7 \mathrm{~nm}$ away from the interface. Spectra further into the GaAs layer show an almost identical shape with spectrum (a). Spectra across the entire GaAs layer extending over a $50 \mathrm{~nm}$ range have been repeatedly measured. The results reveal nearly perfect flat band conditions across the two GaAs layers surrounding the InGaP layer. The GaAs CBM is determined to be at $1.08 \mathrm{eV}$ while the $\mathrm{VBM}$ is $-0.55 \mathrm{eV}$. The observed band gap is thus $1.63 \pm 0.07 \mathrm{eV}$, which is somewhat larger than the known gap of $1.42 \mathrm{eV}$. Spectrum (h) was taken inside the InGaP layer, $6.2 \mathrm{~nm}$ from the interface. The CBM is determined to be at $1.08 \mathrm{eV}$ while the VBM is at $-1.10 \mathrm{eV}$. The observed band gap is thus $2.18 \pm 0.07$ $\mathrm{eV}$, again slightly larger than the known gap of $1.90 \mathrm{eV}$. We attribute these larger gaps to tip-induced band bending in the semiconductor [10]. The difference between the CBM of InGaP and $\mathrm{GaAs}$ is found to be $0.00 \pm 0.03 \mathrm{eV}$. Detailed three-dimensional electrostatics simulations, following Ref. [15] but for the present geometry of an undoped InGaP layer surrounded by doped GaAs layers, indicates a possible change in tip-induced band bending of $0.00-0.06 \mathrm{eV}$ when the tip moves across the interface (as for spectra (a) to (h) of Fig. 3) [16]. Including this result as a correction factor yields a conduction band offset of $0.03 \pm 0.06 \mathrm{eV}$ with InGaP on top (i.e. type I). Results on the other interface (InGaPon-GaAs) are similar, yielding a conduction band offset of $0.05 \pm 0.06 \mathrm{eV}$ (type I).

There are several groups who have reported small conduction band offsets with GSMBE InGaP samples, varying from 0.03 to $0.12 \mathrm{eV}$ (type I) $[3,5,9,17,18,19]$. Froyen et al. [20] theoretically predicted a conduction band offset of $0.12 \mathrm{eV}$ (type I) between GaAs and fully disordered InGaP layer. The averaged result of our study, $0.04 \pm 0.06 \mathrm{eV}$ (type I), is consistent with those previous results. Other groups using predominantly MOCVD report larger conduction band offsets, varying from $0.19 \mathrm{eV}$ to $0.39 \mathrm{eV}$ (e.g. 
Refs. $[2,4]$ ), again type I. This difference between large and small offsets does not appear to be from an ordering effect. Froyen et al. [20] reported a decrease of conduction band offset when InGaP orders, with a type II alignment for fully ordered InGaP. Perhaps the differing results arise from different interface structures formed by the varying growth method and growth conditions. The present study combines nanoscale real-space imaging and energy-space mapping, providing a better understanding of band offset for a welldefined interface. We can confidently say that a small or zero conduction band offset exists when there is a narrow InGaAs-like interface and As/P interchange is not dominant.

In conclusion, InGaP/GaAs heterostructures have been studied by cross-sectional scanning tunneling microscopy and spectroscopy. A random InGaP alloy is observed. It is clearly seen that In atoms incorporate into the following GaAs layer. Both interfaces appear to be InGaAs-like for the growth sequence used here. Spectroscopy measurements reveal a conduction band offset of $0.04 \pm 0.06 \mathrm{eV}$ (type I).

This work was supported by the National Science Foundation, grant DMR-9985898 and by the Deutsche Forschungsgemeinschaft within the framework of "Forschergruppe 394". 


\section{References:}

[1] H. Kroemer, J. Vac. Sci. Technol. B 1, 126 (1983).

[2] M. O. Watanabe, and Y. Ohba, Appl. Phys. Lett. 50, 906 (1987).

[3] J. H. Chen, J. R. Sites, I. L. Spain, M. J. Hafich, and G. Y. Robinson, Appl. Phys. Lett. 58, 744 (1991).

[4] T. W. Lee, P. A. Houston, R. Kumar, X. F. Yang, G. Hill, M. Hopkinson, and P. A. Claxton, Appl. Phys. Lett. 60, 474 (1992).

[5] C. Cai, M. I. Nathan, and T. H. Lim, Appl. Phys. Lett. 74, 720 (1999).

[6] K. Ouchi, and T. Mishima, J. Cryst. Growth 209, 242 (2000).

[7] H. W. M. Salemink, O. Albrektsen, and P. Koenraad, Phys. Rev. B 45, 6946 (1992).

[8] N. Liu, C. K. Shih, J. Geisz, A. Mascarenhas, and J. M. Olson, Appl. Phys. Lett. 73, 1979 (1998).

[9] W. T. Masselink, M. Zachau, T. W. Hickmott, and K. Hendrickson, J. Vac. Sci. Technol. B 10, 966 (1992).

[10] R. M. Feenstra, Phys. Rev. B 50, 4561 (1994).

[11] H. A. McKay, H. Chen, R. M. Feenstra, and P.J. Poole, J. Vac. Sci. Technol. B 21, 18 (2003).

[12] C. Nozaki, Y. Ohba, H. Sugawara, S. Yasuami, and T. Nakanisi, J. Cryst. Growth 93, 406 (1988).

[13] M. Mesrine, J. Massies, E. Vanelle, N. Grandjean, and C. Deparis, Appl. Phys. Lett. 71, 3552 (1997).

[14] C. Y. Tsai, M. Moser, C. Geng, V. Harle, T. Forner, P. Michler, A. Hangleiter, and F. Scholz, J. Crystal Growth 145, 786 (1994).

[15] R. M. Feenstra, J. Vac. Sci. Technol. B 21, 2080 (2003).

[16] Y. Dong, R. M. Feenstra, M. P. Semtsiv and W. T. Masselink, to be published.

[17] T. Kobayashi, K. Taira, F. Nakamura, and H. Kawai, J. Appl. Phys. 65, 4895 (1989).

[18] C. Jelen, S. Slivken, J. Hoff, M. Razeghi, and G. J. Brown, Appl. Phys. Lett. 70, 2173 (1997).

[19] M. A. Haase, M. J. Hafich, and G. Y. Robinson, Appl. Phys. Lett. 58, 616 (1991).

[20] S. Froyen, A. Zunger, and A. Mascarenhas, Appl. Phys. Lett. 68, 2852 (1996). 

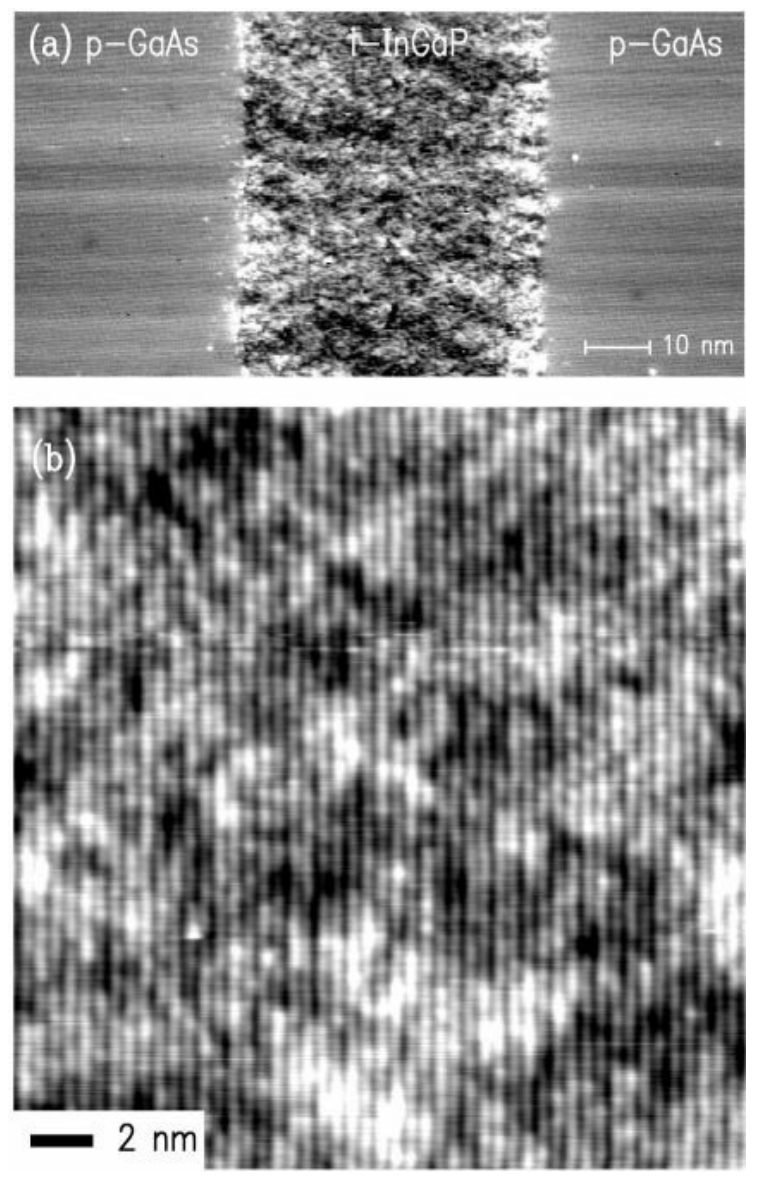

Fig. 1 (a) STM image of p-GaAs/i-InGaP/p-GaAs heterostructure, acquired with sample voltage of $2.5 \mathrm{~V}$. (b) High-resolution image of InGaP layer, acquired with sample voltage of $-2.0 \mathrm{~V}$ and displayed with a gray scale of $0.5 \AA$. Growth direction is from right to left. 


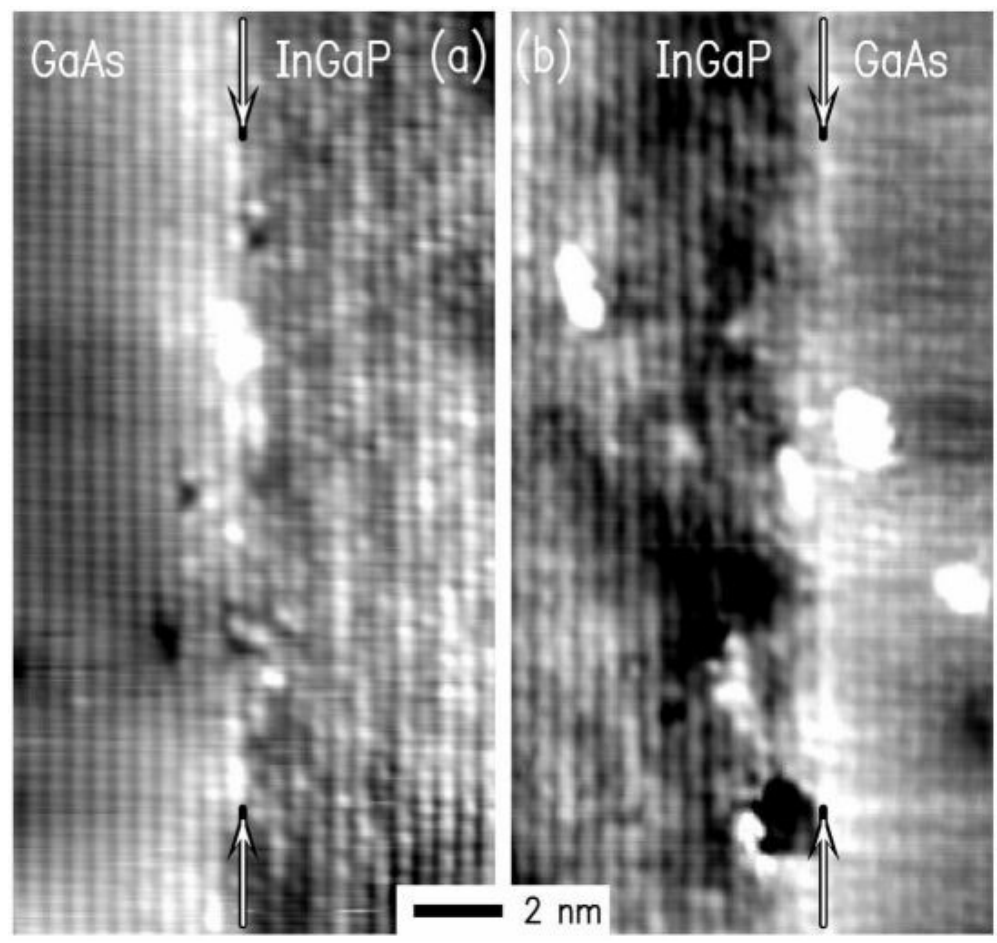

Fig. 2 STM images of (a) GaAs-on-InGaP and (b) InGaP-on-GaAs interface. Both image were acquired with sample voltage of $-2.0 \mathrm{~V}$ and are displayed with gray scales of $0.9 \AA$. Growth direction is from right to left. 

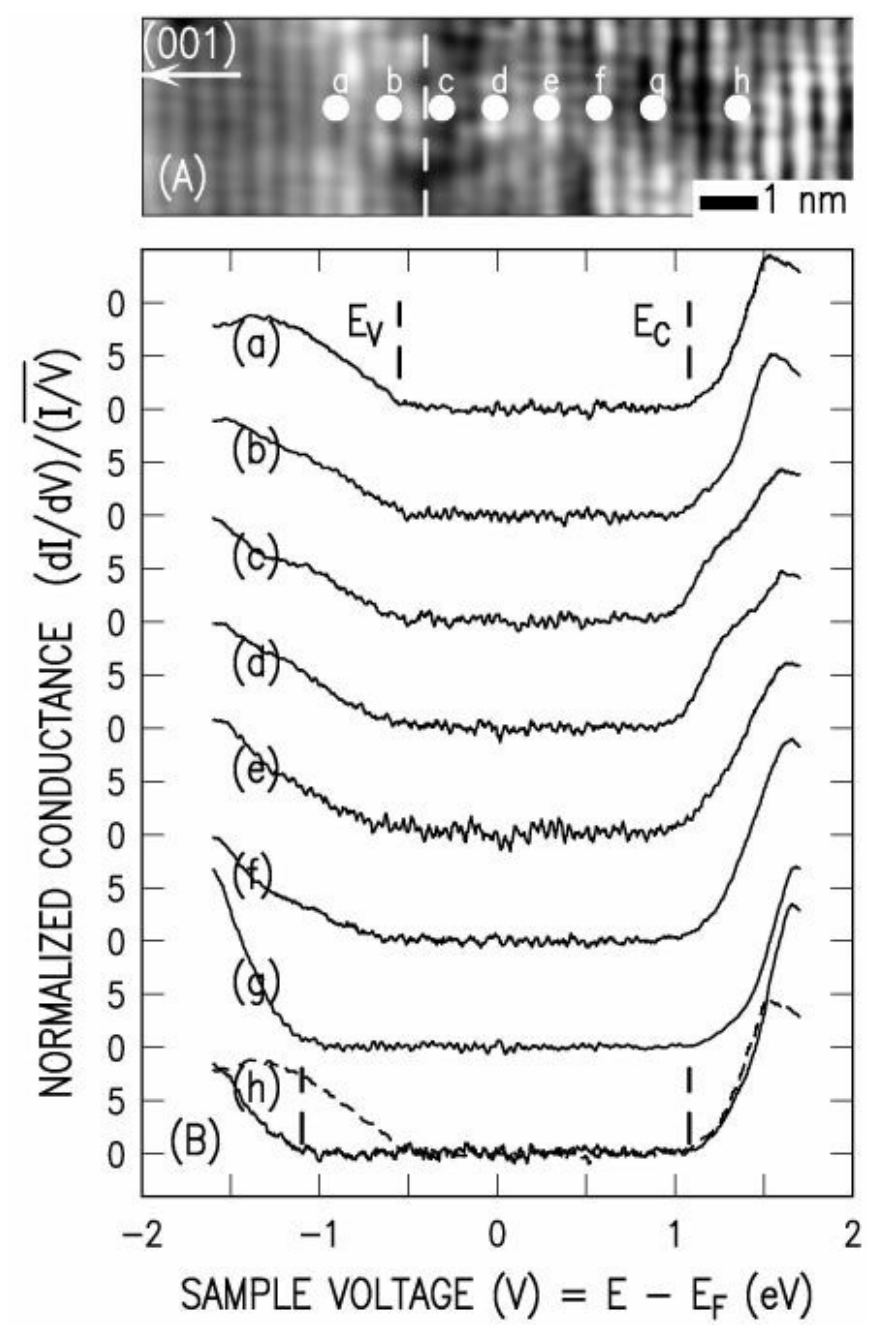

Fig. 3 (A) STM image of GaAs-on-InGaP interface, acquired at a sample voltage of -2.0 V. White circles represent positions where spectra are taken. The dashed line in the left side of image labels the interface, with the InGaP layer being on the right side of the interface and the GaAs layer on the left. (B) Tunneling spectra across the interface. The sample voltage corresponds to the energy of a state relative to the Fermi level. For comparison purposes, spectrum (a) is overlaid as a dashed line on spectrum (h). 